\title{
MAGNETO-THERMOELASTIC WAVES IN A PERFECTLY CONDUCTING ELASTIC HALF-SPACE IN THERMOELASTICITY III
}

\author{
S. K. ROYCHOUDHURI AND NUPUR BANDYOPADHYAY
}

Received 21 April 2005

The propagation of magneto-thermoelastic disturbances in an elastic half-space caused by the application of a thermal shock on the stress-free bounding surface in contact with vacuum is investigated. The theory of thermoelasticity III proposed by Green and Naghdi is used to study the interaction between elastic, thermal, and magnetic fields. Small-time approximations of solutions for displacement, temperature, stress, perturbed magnetic fields both in the vacuum and in the half-space are derived. The solutions for displacement, temperature, stress, perturbed magnetic field in the solid consist of a dilatational wave front with attenuation depending on magneto-thermoelastic coupling and also consists of a part diffusive in nature due to the damping term present in the heat transport equation, while the perturbed field in vacuum represents a wave front without attenuation traveling with Alfv'en acoustic wave speed. Displacement and temperatures are continuous at the elastic wave front, while both the stress and the perturbed magnetic field in the half-space suffer finite jumps at this location. Numerical results for a copper-like material are presented.

\section{Introduction}

Nowacki [11], Kaliski and Nowacki [7] considered magneto-thermoelastic waves in a perfectly electrically conducting elastic half-space in contact with a vacuum due to application of a thermal disturbance on the plane boundary. Both the half-space and the vacuum are supposed to be permeated by an applied primary uniform magnetic field. The study was made neglecting the influence of coupling between the thermal and elastic fields. Later, Massalas and Dalamangas $[9,10]$ studied the same problem taking into account the coupling of strain and temperature fields. Further, the investigation carried out in [9] was extended to TRDTE developed by Green and Lindsay [4], by Roychoudhuri and Chatterjee [3, 16]. Moreover, the problem studied in [9] was also extended to ETE developed by Lord and Shulman [8], by Roychoudhuri and Chatterjee [15], and by Roychoudhuri and Banerjee [14]. Further, Roychoudhuri and Debnath [17], Roychoudhuri $[12,13]$ studied magneto-thermoelastic waves in a rotating solid in the context of ETE.

Copyright (C) 2005 Hindawi Publishing Corporation

International Journal of Mathematics and Mathematical Sciences 2005:20 (2005) 3303-3318

DOI: 10.1155/IJMMS.2005.3303 
Transient magneto-thermoelastic waves in a rotating half-space were also studied in the context of ETE by Chand et al. [2].

Recently, Green and Naghdi $[5,6]$ developed a theory where the characteristics of material response for thermal phenomena are based on three types of constitutive response functions, labeled as types I, II, and III. The nature of these three types of constitutive equations is such that when the respective theories are linearized, type I is the same as the classical heat conduction equation (based on Fourier's law), whereas the linearized version of type II theory accommodates finite thermal wave speed and involves no dissipation of thermal energy. Further, the type III theory involves a thermal damping term. The mixed third-order derivative term appearing in the heat transport equation destroys the wave structure. Accordingly, this equation predicts a non-wave-like heat conduction different from the usual diffusion equation predicted by the conventional parabolic heat equation. This theory admits an infinite speed of thermal propagation. This model admits coupled damped thermoelastic waves. The purpose of the present study is to consider magneto-thermoelastic waves in an elastic half-space in contact with a vacuum due to a thermal shock applied on the stress-free plane boundary in the context of the thermoelasticity theory III. The medium is supposed to be a perfect electrical conductor and both media are permeated by a primary uniform magnetic field parallel to the plane boundary. Short-time solutions for displacement, temperature, stress, perturbed fields in the halfspace and that in the vacuum are derived. The solutions for displacement, temperature, stress, and perturbed field in the solid consist of an elastic wave front with attenuation and a diffusive part due to the damping term present in the heat transport equation. The perturbed magnetic field in vacuum represents a wave front without any attenuation traveling with Alfv'en acoustic wave speed. Displacement and temperature in the half-space are found to be continuous at the elastic wave front, while the stress and the perturbed magnetic field in the solid both experience finite discontinuity at the same location. The finite discontinuities are not constants but decay exponentially with distance from the boundary.

\section{Formulation of the problem and basic equations}

We consider a homogeneous, isotropic, thermally and electrically conducting elastic halfspace $D: x \geq 0$ at a uniform reference temperature $\theta_{0}$ in contact with a vacuum $D^{\prime}: x<0$. We suppose that in both media, there is an initial uniform magnetic field of intensity $\vec{H}_{0}$ acting in the $z$-direction so that $\vec{H}_{0}=\left(0,0, H_{0}\right)$, where $H_{0}$ is a constant. At the instant $t=0+$, we assume that the stress-free plane boundary $x=0$ is suddenly heated to a temperature $T_{0}$ and left in this state.

The thermal shock $T=T_{0} H(t)$, where $H(t)$ is the Heavy-side unit step function, produces in the half-space a magneto-elastic wave which depends on the spatial coordinate $x$ and time $t$. At the same time, an electromagnetic wave is radiated into the vacuum [7].

The simplified linearized equations of electrodynamics of slowly moving continuous media having perfect electrical conductivity are

$$
\vec{\nabla} \times \vec{h}=\frac{4 \pi}{c} \vec{j}, \quad \vec{\nabla} \times \vec{E}=-\frac{\mu_{e}}{c} \frac{\partial \vec{h}}{\partial t}, \quad \vec{\nabla} \cdot \vec{h}=0, \quad \vec{E}=-\frac{\mu_{e}}{c}\left(\frac{\partial \vec{u}}{\partial t} \times \vec{H}_{0}\right),
$$


where $\vec{h}$ and $\vec{E}$ denote perturbations of the magnetic and electric fields, respectively, $\vec{j}$ is the electric current density vector, $\vec{H}_{0}$ is the initial constant magnetic field, $\vec{u}$ is the mechanical displacement vector, $\mu_{e}$ is the magnetic permeability, and $c$ is the velocity of light. The displacement equations of motion in magneto-thermoelasticity are

$$
\mu \nabla^{2} \vec{u}+(\lambda+\mu) \vec{\nabla}(\operatorname{div} \vec{u})-\gamma \vec{\nabla} T+\frac{1}{c}(\vec{j} \times \vec{B})=\rho \ddot{\vec{u}},
$$

where $(\vec{j} \times \vec{B})$ is the electromagnetic body force, $\vec{B}$ is the magnetic induction vector, $\lambda, \mu$ are Lame's constants, $\gamma=(3 \lambda+2 \mu) \alpha_{t}$, $\alpha_{t}$ is the coefficient of linear thermal expansion, $T$ is the temperature increase above the reference temperature $\theta_{0}$, and $\rho$ is the constant mass density.

After linearization,

$$
\vec{j} \times \vec{B}=\vec{j} \times \mu_{e}\left(\vec{H}_{0}+\vec{h}\right) \cong \mu_{e}\left(\vec{j} \times \vec{H}_{0}\right)=\frac{\mu_{e} c}{4 \pi}\left[(\vec{\nabla} \times \vec{h}) \times \vec{H}_{0}\right] .
$$

Equation (2.2), then, after linearization, reduces to

$$
\mu \nabla^{2} \vec{u}+(\lambda+\mu) \vec{\nabla}(\operatorname{div} \vec{u})-\gamma \vec{\nabla} T+\frac{\mu_{e} c}{4 \pi}\left[(\vec{\nabla} \times \vec{h}) \times \vec{H}_{0}\right]=\rho \ddot{\vec{u}} .
$$

The heat transport equation in the theory of thermoelasticity (type III) presented by Green and Naghdi [5] (in absence of heat sources) is

$$
\rho C_{v} \ddot{T}+\gamma \theta_{0} \operatorname{div} \ddot{\vec{u}}=K \nabla^{2} \dot{T}+K^{*} \nabla^{2} T, \quad K^{*}>0,
$$

where $C_{v}$ is the specific heat at constant strain, $K$ is the thermal conductivity, and $K^{*}$ is a material constant characteristic of the theory. It may be noted that the third model represented by (2.5) of Green and Naghdi [5] for heat transport in solids accommodates infinite thermal wave speed due to the presence of third-order mixed derivative term present on the right-hand side of (2.5) and it involves thermal damping. As such, the corresponding thermoelastic model admits coupled damped thermoelastic waves.

Since the disturbances depend on the spatial coordinate $x$ and time $t$, we assume, for one-dimensional deformation,

$$
\vec{u}=[u(x, t), 0,0], \quad T=T(x, t), \quad \vec{h}=\vec{h}(x, t) .
$$

For $\vec{H}_{0}=\left(0,0, H_{0}\right),(2.1)$ reduces to

$$
\begin{aligned}
\vec{E} & =\left(0, \frac{\mu_{e} H_{0}}{c} \dot{u}, 0\right), \\
\frac{\partial \vec{h}}{\partial t} & =\left(0,0,-H_{0} \frac{\partial \dot{u}}{\partial x}\right), \\
\vec{j} & =\left(0,-\frac{c}{4 \pi} \frac{\partial h_{z}}{\partial x}, 0\right) .
\end{aligned}
$$


3306 Magneto-thermoelastic waves in thermoelasticity III

The second equation of (2.7), on integration, yields $h_{z}=-H_{0}(\partial u / \partial x)$ for a perfect conductor.

Equations (2.4) and (2.5), in one-dimensional case, for a perfect conductor simplify to

$$
\begin{gathered}
\left(\lambda+2 \mu+a_{0}^{2} \rho\right) \frac{\partial^{2} u}{\partial x^{2}}-\gamma \frac{\partial T}{\partial x}=\rho \frac{\partial^{2} u}{\partial t^{2}} \\
\rho C_{v} \frac{\partial^{2} T}{\partial t^{2}}+\gamma \theta_{0} \frac{\partial^{3} u}{\partial x \partial t^{2}}=K \frac{\partial^{3} T}{\partial x^{2} \partial t}+K^{*} \frac{\partial^{2} T}{\partial x^{2}}
\end{gathered}
$$

where $a_{0}=\sqrt{\mu_{e} H_{0}^{2} / 4 \pi \rho}$ is the Alfv'en wave velocity of the medium.

The system of Maxwell's equations in vacuum is expressed as

$$
\vec{\nabla} \times \vec{h}^{0}=\frac{1}{c} \frac{\partial \vec{D}^{0}}{\partial t}, \quad \vec{\nabla} \cdot \vec{h}^{0}=0, \quad \vec{\nabla} \cdot \vec{E}^{0}=0 .
$$

where $\vec{h}^{0}$ and $\vec{E}^{0}$ are the perturbed magnetic field and the electric field in vacuum. These give

$$
\vec{\nabla} \times \vec{h}^{0}=\frac{1}{c} \frac{\partial \vec{E}^{0}}{\partial t}, \quad \vec{\nabla} \times \vec{E}^{0}=-\frac{1}{c} \frac{\partial \vec{h}^{0}}{\partial t} .
$$

These equations yield the following equations satisfied by $\vec{E}^{0}$ and $\vec{h}^{0}$ :

$$
\left(\nabla^{2}-\frac{1}{c^{2}} \frac{\partial^{2}}{\partial t^{2}}\right)\left(\vec{E}^{0}, \vec{h}^{0}\right)=0
$$

In one-dimensional case, this reduces to

$$
\left(\frac{\partial^{2}}{\partial x^{\prime 2}}-\frac{1}{c^{2}} \frac{\partial^{2}}{\partial t^{2}}\right)\left(E_{y}^{0}, h_{z}^{0}\right)=0
$$

where $x^{\prime}=-x$.

The components $T_{11}$ and $T_{11}^{0}$ of Maxwell's stress tensors in the elastic medium and in vacuum are given by

$$
T_{11}=-\frac{\mu_{e}}{4 \pi} h_{z} H_{0}, \quad T_{11}^{0}=-\frac{1}{4 \pi} h_{z}^{0} H_{0} .
$$

The total stress in the half-space is composed of Hooke's mechanical stress and Maxwell's stress. Thus, the total stress in the half-space is

$$
\begin{aligned}
\boldsymbol{\sigma}_{11}^{*} & =\boldsymbol{\sigma}_{11}+T_{11}=(\lambda+2 \mu) \frac{\partial u}{\partial x}-\gamma T+T_{11} \\
& =(\lambda+2 \mu) \frac{\partial u}{\partial x}-\gamma T-\frac{\mu_{e}}{4 \pi} h_{z} H_{0} \\
& =\left\{(\lambda+2 \mu)+\frac{\mu_{e}}{4 \pi} H_{0}^{2}\right\} \frac{\partial u}{\partial x}-\gamma T,
\end{aligned}
$$

where $\sigma_{11}$ is the Hooke mechanical stress. 
Boundary conditions. (i) The continuity of total stress composed of thermoelastic and electromagnetic stress across the boundary $x=x^{\prime}=0$ yields

$$
\sigma_{11}+T_{11}=T_{11}^{0} \quad \text { on } x=x^{\prime}=0 .
$$

(ii) The tangential component of $\vec{E}$-field is continuous across $x=x^{\prime}=0$, which leads to

$$
E_{y}=E_{y}^{0} \quad \text { on } x=x^{\prime}=0 .
$$

(iii) The thermal boundary condition on $x=x^{\prime}=0$ gives $T(0, t)=T_{0} H(t)$, where $T_{0}$ is a constant.

We assume that the system is at rest initially and temperature and temperature velocity all vanish initially.

Then

$$
u(x, 0)=\dot{u}(x, 0)=0, \quad T(x, 0)=\dot{T}(x, 0)=0 .
$$

We introduce the following notations and nondimensional variables:

$$
\begin{gathered}
c_{1}^{2}=\frac{\lambda+2 \mu}{\rho}, \quad c_{0}^{2}=a_{0}^{2}+c_{1}^{2}, \quad \kappa=\frac{K}{\rho C_{v}}, \quad \xi=\frac{c_{0} x}{\kappa}, \quad \eta=\frac{c_{0}^{2} t}{\kappa}, \\
U=\frac{c_{0}\left(\lambda+2 \mu+a_{0}^{2} \rho\right)}{\gamma \theta_{0} \kappa} u, \quad \Theta=\frac{T}{\theta_{0}},
\end{gathered}
$$

where $c_{1}$ is the dilatational wave velocity in the half-space. Equations (2.8) then reduce to nondimensional forms as

$$
\begin{gathered}
\frac{\partial^{2} U}{\partial \xi^{2}}-\frac{\partial \Theta}{\partial \xi}=\frac{\partial^{2} U}{\partial \eta^{2}}, \quad \xi>0, \\
\frac{\partial^{2} \Theta}{\partial \eta^{2}}+\varepsilon_{T} \frac{\partial^{3} U}{\partial \xi \partial \eta^{2}}=\frac{\partial^{3} \Theta}{\partial \eta \partial \xi^{2}}+C_{T}^{2} \frac{\partial^{2} \Theta}{\partial \xi^{2}}, \quad \xi>0,
\end{gathered}
$$

where $\varepsilon_{T}=\gamma^{2} \theta_{0} / \rho^{2} C_{\nu} c_{0}^{2}$ is the magneto-thermoelastic coupling, which reduces to $\varepsilon$ the thermoelastic coupling constant for $H_{0}=0$.

Equations (2.19)-(2.20) admit damped magneto-thermoelastic wave solutions in the half-space. Here $C_{T}=c_{3} / c_{0}$, where $c_{3}=\sqrt{K^{*} / \rho C_{v}}$ and $C_{T}$ is the nondimensional finite thermal wave speed corresponding to $c_{3}$ which is the finite thermal wave speed of GN model II.

In $D^{\prime}: x^{\prime}>0$, that is, $x<0$, the equation satisfied by $h_{z}^{0}$ reduces to

$$
\left(\frac{\partial^{2}}{\partial \xi^{\prime 2}}-\beta^{2} \frac{\partial^{2}}{\partial \eta^{2}}\right) h_{z}^{0}=0, \quad \text { for } \xi^{\prime}>0, \xi^{\prime}=-\xi
$$

where $\beta=c_{0} / c$ and $1 / \beta$ is the Alfv'en acoustic wave velocity of the medium. 
Further, the boundary condition for continuity of total stress across $\xi=\xi^{\prime}=0$ in nondimensional form reduces to

$$
\frac{\partial U}{\partial \xi}-\Theta+\beta_{1} h_{z}^{0}=0 \quad \text { on } \xi=\xi^{\prime}=0,
$$

where $\beta_{1}=H_{0} / 4 \pi \gamma \theta_{0}$.

The condition of continuity of $\vec{E}$-field across $x=x^{\prime}=0$ reduces to $E_{y}=E_{y}^{0}$ which, by the help of (2.7), (2.9), and (2.12), yields, in nondimensional form, the following equation:

$$
\beta_{2} \frac{\partial^{2} U}{\partial \eta^{2}}-\frac{\partial h_{z}^{0}}{\partial \xi^{\prime}}=0 \quad \text { on } \xi=\xi^{\prime}=0
$$

where $\beta_{2}=\mu_{e} H_{0} \gamma \theta_{0} / \rho c^{2}$.

Lastly, the thermal boundary condition gives

$$
\Theta(0, \eta)=\frac{T_{0}}{\theta_{0}} H(\eta)
$$

The initial conditions are

$$
U(\xi, 0)=\frac{\partial U(\xi, 0)}{\partial \eta}=0, \quad \Theta(\xi, 0)=\frac{\partial \Theta(\xi, 0)}{\partial \eta}=0 .
$$

The nondimensional total stress in the half-space is obtained from (2.14) as

$$
\boldsymbol{\sigma}_{11}^{\prime}=\frac{\sigma_{11}^{*}}{\gamma T_{0}}=\frac{\partial U}{\partial \xi}-\Theta
$$

The perturbed magnetic field in the half-space is $h_{z}=-H_{0}(\partial u / \partial x)$ which in nondimensional form reduces to

$$
h_{z}^{\prime}=-\frac{\partial U}{\partial \xi}
$$

where $h_{z}^{\prime}=\left(\rho c_{0}^{2} / H_{0} \gamma \theta_{0}\right) h_{z}=$ nondimensional form of $h_{z}$.

It may be observed that (2.19) and (2.21) are fully hyperbolic but the mixed derivative term on the right-hand side of (2.20) destroys the wave structure. In fact, this equation predicts a non-wave-like heat conduction different from the usual diffusion equation predicted by conventional parabolic heat equation. It is therefore expected that the solutions of the coupled equations (2.19)-(2.20) with coupled boundary conditions (2.22)-(2.23) should be composed of a wave part (dilatational wave) and a diffusive part due to the presence of the thermal damping term in the heat transport equation. As (2.21) (hyperbolic type) uncouples from the system, its solution will be composed of a wave part traveling with Alfv'en acoustic wave speed in vacuum. 
Solution of the problem in the Laplace transform domain. We introduce a potential function $\phi$ defined by

$$
U=\frac{\partial \phi}{\partial \xi}
$$

Then (2.19), on integrating with respect to $\xi$, yields

$$
\Theta(\xi, \eta)=\left(\frac{\partial^{2}}{\partial \xi^{2}}-\frac{\partial^{2}}{\partial \eta^{2}}\right) \phi, \quad \xi>0 .
$$

Equation (2.20) becomes

$$
\frac{\partial^{2} \Theta}{\partial \eta^{2}}+\varepsilon_{T} \frac{\partial^{4} \phi}{\partial \xi^{2} \partial \eta^{2}}=\frac{\partial^{3} \Theta}{\partial \eta \partial \xi^{2}}+C_{T}^{2} \frac{\partial^{2} \Theta}{\partial \xi^{2}}, \quad \xi>0 .
$$

Taking Laplace transform of (2.29), (2.30), and (2.21) and using the initial conditions, we obtain

$$
\begin{gathered}
\bar{\Theta}(\xi, s)=\left(\frac{d^{2}}{d \xi^{2}}-s^{2}\right) \bar{\phi}, \quad \xi>0, \\
{\left[\left(C_{T}^{2}+s\right) \frac{d^{2}}{d \xi^{2}}-s^{2}\right] \bar{\Theta}=\varepsilon_{T} s^{2} \frac{d^{2} \bar{\phi}}{d \xi^{2}}, \quad \xi>0,} \\
\frac{d^{2} \overline{h_{z}^{0}}}{d \xi^{2}}=\beta^{2} s^{2} \overline{h_{z}^{0}}, \quad \xi^{\prime}>0
\end{gathered}
$$

where $s$ is the Laplace transform parameter. Further (2.26)-(2.27) in the Laplace transform domain become

$$
\begin{gathered}
\overline{\sigma_{11}^{\prime}}=\frac{d \bar{U}}{d \xi}-\bar{\Theta}=\frac{d^{2} \bar{\phi}}{d \xi^{2}}-\bar{\Theta}, \quad \xi>0, \\
\overline{h_{z}^{\prime}}=-\frac{d \bar{U}}{d \xi}=-\frac{d^{2} \bar{\phi}}{d \xi^{2}}, \quad \xi>0 .
\end{gathered}
$$

The boundary conditions (2.22)-(2.24) in the Laplace transform domain reduce to the following:

$$
\begin{gathered}
\frac{d^{2} \bar{\phi}}{d \xi^{2}}-\bar{\Theta}+\beta_{1} \overline{h_{z}^{0}}=0 \quad \text { on } \xi=\xi^{\prime}=0, \\
\beta_{2} s^{2} \frac{d \bar{\phi}}{d \xi}-\frac{d \overline{h_{z}^{0}}}{d \xi^{\prime}}=0 \quad \text { on } \xi=\xi^{\prime}=0, \\
\bar{\Theta}=\frac{T_{0}}{\theta_{0}} \frac{1}{s} \quad \text { on } \xi=0 .
\end{gathered}
$$

Elimination of $\bar{\Theta}$ from (2.31) and (2.32) yields

$$
\left[\left(C_{T}^{2}+s\right) \frac{d^{4}}{d \xi^{4}}-\left(1+\varepsilon_{T}+C_{T}^{2}+s\right) s^{2} \frac{d^{2}}{d \xi^{2}}+s^{4}\right] \bar{\phi}=0 .
$$


3310 Magneto-thermoelastic waves in thermoelasticity III

The general solution of the above equation, vanishing as $\xi \rightarrow \infty$, is given by

$$
\bar{\varphi}(\xi, s)=A_{1} \exp \left(-\lambda_{1} \xi\right)+B_{1} \exp \left(-\lambda_{2} \xi\right), \quad \xi>0,
$$

where $\lambda_{1,2}^{2}$ are the roots of the quadratic equation

$$
\left(C_{T}^{2}+s\right) \lambda^{4}-\left(1+\varepsilon_{T}+C_{T}^{2}+s\right) s^{2} \lambda^{2}+s^{4}=0 .
$$

Hence,

$$
\begin{aligned}
& \lambda_{1}=s\left[\frac{(a+s)+\sqrt{(a+s)^{2}-4\left(C_{T}^{2}+s\right)}}{2\left(C_{T}^{2}+s\right)}\right]^{1 / 2}, \\
& \lambda_{2}=s\left[\frac{(a+s)-\sqrt{(a+s)^{2}-4\left(C_{T}^{2}+s\right)}}{2\left(C_{T}^{2}+s\right)}\right]^{1 / 2},
\end{aligned}
$$

where $a=1+\varepsilon_{T}+C_{T}^{2}$.

From (2.31) and (2.37), we obtain

$$
\bar{\Theta}(\xi, s)=A_{1}\left(\lambda_{1}^{2}-s^{2}\right) \exp \left(-\lambda_{1} \xi\right)+B_{1}\left(\lambda_{2}^{2}-s^{2}\right) \exp \left(-\lambda_{2} \xi\right), \quad \xi>0 .
$$

Further, (2.33) yields

$$
\overline{h_{z}^{0}}=C_{1} \exp \left(-s \beta \xi^{\prime}\right), \quad \xi^{\prime}>0
$$

The constants $A_{1}, B_{1}, C_{1}$ are obtained with the help of the boundary conditions (2.35).

Hence,

$$
\begin{gathered}
\bar{\varphi}(\xi, s)=\frac{T_{0}}{\theta_{0} s} \frac{\left(s \beta+\beta_{1} \beta_{2} \lambda_{2}\right) e^{-\lambda_{1} \xi}-\left(s \beta+\beta_{1} \beta_{2} \lambda_{1}\right) e^{-\lambda_{2} \xi}}{\left(\lambda_{1}-\lambda_{2}\right)\left\{\beta_{1} \beta_{2} s^{2}+\beta\left(\lambda_{1}+\lambda_{2}\right) s+\beta_{1} \beta_{2} \lambda_{1} \lambda_{2}\right\}}, \quad \xi>0, \\
\bar{U}(\xi, s)=\frac{T_{0}}{\theta_{0} s} \frac{\lambda_{2}\left(s \beta+\beta_{1} \beta_{2} \lambda_{1}\right) e^{-\lambda_{2} \xi}-\lambda_{1}\left(s \beta+\beta_{1} \beta_{2} \lambda_{2}\right) e^{-\lambda_{1} \xi}}{\left(\lambda_{1}-\lambda_{2}\right)\left\{\beta_{1} \beta_{2} s^{2}+\beta\left(\lambda_{1}+\lambda_{2}\right) s+\beta_{1} \beta_{2} \lambda_{1} \lambda_{2}\right\}}, \quad \xi>0, \\
\bar{\Theta}(\xi, s)=\frac{T_{0}}{\theta_{0} s} \frac{\left(\lambda_{1}^{2}-s^{2}\right)\left(s \beta+\beta_{1} \beta_{2} \lambda_{2}\right) e^{-\lambda_{1} \xi}-\left(\lambda_{2}^{2}-s^{2}\right)\left(s \beta+\beta_{1} \beta_{2} \lambda_{1}\right) e^{-\lambda_{2} \xi}}{\left(\lambda_{1}-\lambda_{2}\right)\left\{\beta_{1} \beta_{2} s^{2}+\beta\left(\lambda_{1}+\lambda_{2}\right) s+\beta_{1} \beta_{2} \lambda_{1} \lambda_{2}\right\}}, \quad \xi>0, \\
\overline{h_{z}^{\prime}}(\xi, s)=\frac{T_{0}}{\theta_{0} s} \frac{\lambda_{2}^{2}\left(s \beta+\beta_{1} \beta_{2} \lambda_{1}\right) e^{-\lambda_{2} \xi}-\lambda_{1}^{2}\left(s \beta+\beta_{1} \beta_{2} \lambda_{2}\right) e^{-\lambda_{1} \xi}}{\left(\lambda_{1}-\lambda_{2}\right)\left\{\beta_{1} \beta_{2} s^{2}+\beta\left(\lambda_{1}+\lambda_{2}\right) s+\beta_{1} \beta_{2} \lambda_{1} \lambda_{2}\right\}}, \quad \xi>0, \\
\overline{h_{z}^{0}}\left(\xi^{\prime}, s\right)=T_{0} \frac{T_{2} e^{-s \beta \xi^{\prime}}}{\beta_{1} \beta_{2} s^{2}+\beta\left(\lambda_{1}+\lambda_{2}\right) s+\beta_{1} \beta_{2} \lambda_{1} \lambda_{2}}, \quad \xi<0, \\
\overline{\sigma_{11}^{\prime}}(\xi, s)=\frac{T_{0}}{\theta_{0}} \frac{s\left(s \beta+\beta_{1} \beta_{2} \lambda_{2}\right) e^{-\lambda_{1} \xi}-s\left(s \beta+\beta_{1} \beta_{2} \lambda_{1}\right) e^{-\lambda_{2} \xi}}{\left(\lambda_{1}-\lambda_{2}\right)\left\{\beta_{1} \beta_{2} s^{2}+\beta\left(\lambda_{1}+\lambda_{2}\right) s+\beta_{1} \beta_{2} \lambda_{1} \lambda_{2}\right\}}, \quad \xi>0 .
\end{gathered}
$$


As the Laplace inversions are very much complicated due to the presence of square root sign in (2.40), we concentrate on solutions for small times only. We make use of Abel's theorem $\lim _{t \rightarrow 0} f(t)=\lim _{s \rightarrow \infty}\{s \bar{f}(s)\}$, that is, small values of time correspond to large values of the parameter $s$. Thus expanding $\lambda_{1,2}$ in ascending powers of $1 / s$ to a few terms, we have

$$
\lambda_{1} \cong \alpha+s+\frac{\alpha_{1}}{s}, \quad \lambda_{2} \cong \sqrt{s}+\frac{\beta_{0}}{\sqrt{s}}+\frac{\gamma_{0}}{s \sqrt{s}} \text { for large } s
$$

where

$$
\begin{gathered}
\alpha=\frac{\varepsilon_{T}}{2}, \quad \alpha_{1}=\frac{4 \varepsilon_{T}\left(1-C_{T}^{2}\right)-\varepsilon_{T}^{2}}{8}, \\
\beta_{0}=-\frac{\varepsilon_{T}+C_{T}^{2}}{2}, \quad \gamma_{0}=\frac{2 \varepsilon_{T} C_{T}^{2}+3 C_{T}^{4}-\varepsilon_{T}^{2}}{8} .
\end{gathered}
$$

Using the approximations (2.44) for large $s$, we have

$$
\begin{aligned}
& \bar{\varphi}(\xi, s) \cong \frac{T_{0}}{\theta_{0} s^{3}} \frac{\beta}{\beta+\beta_{1} \beta_{2}} \exp (-\alpha \xi)\left[1-\frac{a_{2}}{s}+\frac{p_{0}}{\sqrt{s}}\right] \exp (-s \xi) \\
& -\frac{T_{0}}{\theta_{0} s^{3}}\left[1+\frac{p_{0}^{\prime}-a_{2}}{s}-\frac{a_{3}}{s^{3 / 2}}\right] \exp (-\sqrt{s} \xi), \quad \xi>0, \\
& \bar{U}(\xi, s) \cong \frac{T_{0}}{\theta_{0}} \frac{\beta}{\beta+\beta_{1} \beta_{2}} \exp (-\alpha \xi)\left[-\frac{1}{s^{2}}+\frac{a_{2}-\alpha}{s^{3}}-\frac{p_{0}}{s^{2} \sqrt{s}}\right] \exp (-s \xi) \\
& +\frac{T_{0}}{\theta_{0}}\left[\frac{1}{s^{5 / 2}}+\frac{p_{0}^{\prime}-a_{2}}{s^{7 / 2}}\right] \exp (-\sqrt{s} \xi), \quad \xi>0, \\
& \bar{\Theta}(\xi, s) \cong \frac{T_{0}}{\theta_{0}} \frac{\beta}{\beta+\beta_{1} \beta_{2}} \exp (-\alpha \xi)\left[\frac{2 \alpha}{s^{2}}+\frac{k_{0}}{s^{2} \sqrt{s}}-\frac{2 \alpha a_{2}}{s^{3}}\right] \exp (-s \xi) \\
& +\frac{T_{0}}{\theta_{0}}\left[\frac{1}{s}-\frac{k_{0}^{\prime}+a_{2}}{s^{2}}-\frac{a_{3}}{s^{5 / 2}}\right] \exp (-\sqrt{s} \xi), \quad \xi>0, \\
& \overline{h_{z}^{\prime}}(\xi, s)=\frac{T_{0}}{\theta_{0}} \frac{\beta}{\beta+\beta_{1} \beta_{2}} \exp (-\alpha \xi)\left[-\frac{1}{s}-\frac{p_{0}}{s \sqrt{s}}+\frac{a_{2}-2 \alpha}{s^{2}}-\frac{2 \alpha p_{0}}{s^{2} \sqrt{s}}\right] \exp (-s \xi) \\
& +\frac{T_{0}}{\theta_{0}}\left[\frac{1}{s^{2}}+\frac{p_{0}^{\prime}-a_{2}}{s^{3}}-\frac{a_{3}}{s^{7 / 2}}\right] \exp (-\sqrt{s} \xi), \quad \xi>0, \\
& \overline{h_{z}^{0}}\left(\xi^{\prime}, s\right)=\frac{T_{0}}{\theta_{0}} \frac{\beta_{2}}{\beta+\beta_{1} \beta_{2}}\left[\frac{1}{s^{2}}-\frac{a_{2}}{s^{3}}-\frac{a_{3}}{s^{7 / 2}}\right] \exp \left(-s \beta \xi^{\prime}\right), \quad \xi<0, \\
& \overline{\boldsymbol{\sigma}_{11}^{\prime}}(\xi, s) \cong \frac{T_{0}}{\theta_{0}} \frac{\beta_{2}}{\beta+\beta_{1} \beta_{2}} \exp (-\alpha \xi)\left[\frac{1}{s}+\frac{p_{0}}{s \sqrt{s}}-\frac{a_{2}}{s^{2}}+\frac{\alpha p_{0}-k_{0}}{s^{2} \sqrt{s}}\right] \exp (-s \xi) \\
& +\frac{T_{0}}{\theta_{0}}\left[-\frac{1}{s}+\frac{k_{0}^{\prime}+a_{2}-1}{s^{2}}+\frac{a_{3}}{s^{5 / 2}}\right] \exp (-\sqrt{s} \xi), \quad \xi>0 .
\end{aligned}
$$


3312 Magneto-thermoelastic waves in thermoelasticity III

We note the following results after simplification:

$$
\begin{gathered}
\beta=\frac{c_{0}}{c}, \quad \beta_{1}=\frac{H_{0}}{4 \pi \gamma \theta_{0}}, \quad \beta_{2}=\frac{\mu_{e} H_{0} \gamma \theta_{0}}{\rho c^{2}}, \quad a_{2}=\frac{2\left(\varepsilon_{T}-1\right)+\left(\varepsilon_{T}-2\right) \beta_{3}}{2\left(1+\beta_{3}\right)}, \\
p_{0}=\beta_{3}=\frac{\beta_{1} \beta_{2}}{\beta}, \quad p_{0}^{\prime}=\frac{\alpha \beta_{1} \beta_{2}}{\beta+\beta_{1} \beta_{2}}, \quad p_{0}^{\prime}-a_{2}=\frac{\beta_{3}-\varepsilon_{T}+1}{1+\beta_{3}}, \quad a_{3}=\frac{\varepsilon_{T} \beta_{3}}{1+\beta_{3}}, \quad \alpha=\frac{\varepsilon_{T}}{2}, \\
a_{2}-\alpha=\frac{\varepsilon_{T}-2-2 \beta_{3}}{2\left(1+\beta_{3}\right)}, \quad k_{0}=\varepsilon_{T} \beta_{3}, \quad 2 \alpha a_{2}=\frac{2 \varepsilon_{T}\left(\varepsilon_{T}-1\right)+\varepsilon_{T}\left(\varepsilon_{T}-2\right) \beta_{3}}{2\left(1+\beta_{3}\right)}, \\
k_{0}^{\prime}=\frac{\beta+\beta_{1} \beta_{2}(1-\alpha)}{\beta+\beta_{1} \beta_{2}}, \quad k_{0}^{\prime}+a_{2}=\frac{\varepsilon_{T}}{1+\beta_{3}}, \quad \alpha p_{0}-k_{0}=-\frac{\varepsilon_{T} \beta_{3}}{2}, \\
k_{0}^{\prime}+a_{2}-1=\frac{\varepsilon_{T}-1-\beta_{3}}{1+\beta_{3}}, \quad a_{2}-2 \alpha=-\frac{2\left(1+\beta_{3}\right)+\varepsilon_{T} \beta_{3}}{2\left(1+\beta_{3}\right)}, \quad 2 \alpha p_{0}=\varepsilon_{T} \beta_{3} .
\end{gathered}
$$

We then obtain the final expressions of $\bar{\varphi}, \bar{U}, \bar{\Theta}, \overline{h_{z}^{\prime}}, \overline{h_{z}^{0}}, \overline{\sigma_{11}^{\prime}}$ in the following forms in ascending powers of $1 / s$ :

$$
\begin{aligned}
& \bar{\varphi}(\xi, s) \cong \frac{T_{0}}{\theta_{0} s^{3}} \exp \left(-\frac{\varepsilon_{T}}{2} \xi\right)\left[\frac{1}{1+\beta_{3}}-\frac{2\left(\varepsilon_{T}-1\right)+\left(\varepsilon_{T}-2\right) \beta_{3}}{2\left(1+\beta_{3}\right)^{2}} \frac{1}{s}+\frac{\beta_{3}}{1+\beta_{3}} \frac{1}{\sqrt{s}}\right] \exp (-s \xi) \\
& -\frac{T_{0}}{\theta_{0} s^{3}}\left[1+\frac{\beta_{3}-\varepsilon_{T}+1}{1+\beta_{3}} \frac{1}{s}-\frac{\varepsilon_{T} \beta_{3}}{1+\beta_{3}} \frac{1}{s^{3 / 2}}\right] \exp (-\sqrt{s} \xi), \\
& \bar{U}(\xi, s) \cong \frac{T_{0}}{\theta_{0}} \exp \left(-\frac{\varepsilon_{T}}{2} \xi\right)\left[-\frac{1}{1+\beta_{3}} \frac{1}{s^{2}}-\frac{\beta_{3}}{1+\beta_{3}} \frac{1}{s^{2} \sqrt{s}}+\frac{\varepsilon_{T}-2-2 \beta_{3}}{2\left(1+\beta_{3}\right)^{2}} \frac{1}{s^{3}}\right] \exp (-s \xi) \\
& +\frac{T_{0}}{\theta_{0}}\left[\frac{1}{s^{5 / 2}}+\frac{\beta_{3}-\varepsilon_{T}+1}{1+\beta_{3}} \frac{1}{s^{7 / 2}}\right] \exp (-\sqrt{s} \xi) \\
& \bar{\Theta}(\xi, s) \cong \frac{T_{0}}{\theta_{0}} \exp \left(-\frac{\varepsilon_{T}}{2} \xi\right)\left[\frac{\varepsilon_{T}}{1+\beta_{3}} \frac{1}{s^{2}}+\frac{\varepsilon_{T} \beta_{3}}{1+\beta_{3}} \frac{1}{s^{2} \sqrt{s}}-\frac{2 \varepsilon_{T}\left(\varepsilon_{T}-1\right)+\varepsilon_{T}\left(\varepsilon_{T}-2\right) \beta_{3}}{2\left(1+\beta_{3}\right)^{2}} \frac{1}{s^{3}}\right] \exp (-s \xi) \\
& -\frac{T_{0}}{\theta_{0}}\left[\frac{1}{s}-\frac{\varepsilon_{T}}{1+\beta_{3}} \frac{1}{s^{2}}-\frac{\varepsilon_{T} \beta_{3}}{1+\beta_{3}} \frac{1}{s^{5 / 2}}\right] \exp (-\sqrt{s} \xi) \\
& \overline{h_{z}^{\prime}}(\xi, s)=\frac{T_{0}}{\theta_{0}}\left[-\frac{1}{1+\beta_{3}} \frac{1}{s}-\frac{\beta_{3}}{1+\beta_{3}} \frac{1}{s \sqrt{s}}-\frac{2\left(1+\beta_{3}\right)+\varepsilon_{T} \beta_{3}}{2\left(1+\beta_{3}\right)^{2}} \frac{1}{s^{2}}-\frac{\varepsilon_{T} \beta_{3}}{1+\beta_{3}} \frac{1}{s^{2} \sqrt{s}}\right] \exp (-s \xi) \\
& +\frac{T_{0}}{\theta_{0}}\left[\frac{1}{s^{2}}+\frac{\beta_{3}-\varepsilon_{T}+1}{1+\beta_{3}} \frac{1}{s^{3}}-\frac{\varepsilon_{T} \beta_{3}}{1+\beta_{3}} \frac{1}{s^{7 / 2}}\right] \exp (-\sqrt{s} \xi)
\end{aligned}
$$




$$
\begin{aligned}
\overline{h_{z}^{0}}\left(\xi^{\prime}, s\right) \cong & \frac{T_{0}}{\theta_{0}} \frac{\beta_{2}}{\beta\left(1+\beta_{3}\right)}\left[\frac{1}{s^{2}}-\frac{2\left(\varepsilon_{T}-1\right)+\left(\varepsilon_{T}-2\right) \beta_{3}}{2\left(1+\beta_{3}\right)} \frac{1}{s^{3}}-\frac{\varepsilon_{T} \beta_{3}}{1+\beta_{3}} \frac{1}{s^{7 / 2}}\right] \exp \left(-s \beta \xi^{\prime}\right), \\
\overline{\sigma_{11}^{\prime}}(\xi, s) \cong & \frac{T_{0}}{\theta_{0}} \exp \left(-\frac{\varepsilon_{T}}{2} \xi\right)\left[\frac{1}{1+\beta_{3}} \frac{1}{s}+\frac{\beta_{3}}{1+\beta_{3}} \frac{1}{s \sqrt{s}}-\frac{2\left(\varepsilon_{T}-1\right)+\left(\varepsilon_{T}-2\right) \beta_{3}}{2\left(1+\beta_{3}\right)^{2}} \frac{1}{s^{2}}\right. \\
& \left.-\frac{\varepsilon_{T} \beta_{3}}{2\left(1+\beta_{3}\right)} \frac{1}{s^{2} \sqrt{s}}\right] \exp (-s \xi) \\
+ & \frac{T_{0}}{\theta_{0}}\left[-\frac{1}{s}+\frac{\varepsilon_{T}-1-\beta_{3}}{1+\beta_{3}} \frac{1}{s^{2}}+\frac{\varepsilon_{T} \beta_{3}}{1+\beta_{3}} \frac{1}{s^{5 / 2}}\right] \exp (-\sqrt{s} \xi) .
\end{aligned}
$$

Taking inverse of Laplace transforms, we obtain the following small-time solutions of $U, \Theta, h_{z}^{\prime}, h_{z}^{0}, \sigma_{11}$ :

$$
\begin{aligned}
& U(\xi, \eta) \cong \frac{T_{0}}{\theta_{0}} \exp \left(-\frac{\varepsilon_{T}}{2} \xi\right)\left[-\frac{1}{1+\beta_{3}}(\eta-\xi)-\frac{\beta_{3}}{1+\beta_{3}} \frac{4}{3 \sqrt{\pi}}(\eta-\xi)^{3 / 2}\right. \\
& \left.+\frac{\varepsilon_{T}-2-2 \beta_{3}}{2\left(1+\beta_{3}\right)^{2}} \frac{(\eta-\xi)^{2}}{2 !}\right] H(\eta-\xi) \\
& +\frac{T_{0}}{\theta_{0}}\left[(4 \eta)^{3 / 2} i^{3} \operatorname{erf} c\left(\frac{\xi}{2 \sqrt{\eta}}\right)+\frac{\beta_{3}-\varepsilon_{T}+1}{1+\beta_{3}}(4 \eta)^{5 / 2} i^{5} \operatorname{erf} c\left(\frac{\xi}{2 \sqrt{\eta}}\right)\right], \\
& \Theta(\xi, \eta) \cong \frac{T_{0}}{\theta_{0}} \exp \left(-\frac{\varepsilon_{T}}{2} \xi\right)\left[\frac{\varepsilon_{T}}{1+\beta_{3}}(\eta-\xi)+\frac{\varepsilon_{T} \beta_{3}}{1+\beta_{3}} \frac{4}{3 \sqrt{\pi}}(\eta-\xi)^{3 / 2}\right. \\
& \left.-\frac{2 \varepsilon_{T}\left(\varepsilon_{T}-1\right)+\varepsilon_{T}\left(\varepsilon_{T}-2\right) \beta_{3}}{2\left(1+\beta_{3}\right)^{2}} \frac{(\eta-\xi)^{2}}{2 !}\right] H(\eta-\xi) \\
& +\frac{T_{0}}{\theta_{0}}\left[\operatorname{erf} c\left(\frac{\xi}{2 \sqrt{\eta}}\right)-\frac{\varepsilon_{T}}{1+\beta_{3}}(4 \eta) i^{2} \operatorname{erf} c\left(\frac{\xi}{2 \sqrt{\eta}}\right)-\frac{\varepsilon_{T} \beta_{3}}{1+\beta_{3}}(4 \eta)^{3 / 2} i^{3} \operatorname{erf} c\left(\frac{\xi}{2 \sqrt{\eta}}\right)\right], \\
& h_{z}^{\prime}(\xi, \eta)=\frac{T_{0}}{\theta_{0}} \exp \left(-\frac{\varepsilon_{T}}{2} \xi\right)\left[-\frac{1}{1+\beta_{3}}-\frac{\beta_{3}}{1+\beta_{3}} \frac{2}{\sqrt{\pi}}(\eta-\xi)^{1 / 2}\right. \\
& \left.-\frac{2\left(1+\beta_{3}\right)+\varepsilon_{T} \beta_{3}}{2\left(1+\beta_{3}\right)^{2}}(\eta-\xi)-\frac{\varepsilon_{T} \beta_{3}}{1+\beta_{3}} \frac{4}{3 \sqrt{\pi}}(\eta-\xi)^{3 / 2}\right] H(\eta-\xi) \\
& +\frac{T_{0}}{\theta_{0}}\left[(4 \eta) i^{2} \operatorname{erf} c\left(\frac{\xi}{2 \sqrt{\eta}}\right)+\frac{\beta_{3}-\varepsilon_{T}+1}{1+\beta_{3}}(4 \eta)^{2} i^{4} \operatorname{erf} c\left(\frac{\xi}{2 \sqrt{\eta}}\right)\right. \\
& \left.-\frac{\varepsilon_{T} \beta_{3}}{1+\beta_{3}}(4 \eta)^{5 / 2} i^{5} \operatorname{erf} c\left(\frac{\xi}{2 \sqrt{\eta}}\right)\right],
\end{aligned}
$$


3314 Magneto-thermoelastic waves in thermoelasticity III

$$
\begin{aligned}
& h_{z}^{0}\left(\xi^{\prime}, \eta\right) \cong \frac{T_{0}}{\theta_{0}} \frac{\beta_{2}}{\beta\left(1+\beta_{3}\right)}\left[\left(\eta-\beta \xi^{\prime}\right)-\frac{2\left(\varepsilon_{T}-1\right)+\left(\varepsilon_{T}-2\right) \beta_{3}}{2\left(1+\beta_{3}\right)} \frac{\left(\eta-\beta \xi^{\prime}\right)^{2}}{2 !}\right. \\
&\left.-\frac{\varepsilon_{T} \beta_{3}}{1+\beta_{3}} \frac{8}{15 \sqrt{\pi}}\left(\eta-\beta \xi^{\prime}\right)^{5 / 2}\right] H\left(\eta-\beta \xi^{\prime}\right) \\
& \sigma_{11}(\xi, \eta) \cong \frac{T_{0}}{\theta_{0}} \exp \left(-\frac{\varepsilon_{T}}{2} \xi\right)\left[\frac{1}{1+\beta_{3}}+\frac{\beta_{3}}{1+\beta_{3}} \frac{2}{\sqrt{\pi}}(\eta-\xi)^{1 / 2}-\frac{2\left(\varepsilon_{T}-1\right)+\left(\varepsilon_{T}-2\right) \beta_{3}}{2\left(1+\beta_{3}\right)^{2}}(\eta-\xi)\right. \\
&+\frac{T_{0}}{\theta_{0}}\left[-\frac{\varepsilon_{T} \beta_{3}}{2\left(1+\beta_{3}\right)} \frac{4}{3 \sqrt{\pi}}(\eta-\xi)^{3 / 2}\right] H(\eta-\xi) \\
&+\frac{\varepsilon_{T} \beta_{3}}{1+\beta_{3}}(4 \eta)^{3 / 2} i^{3} \operatorname{erf} c\left(\frac{\xi}{2 \sqrt{\eta}}\right)+\frac{\varepsilon_{T}-1-\beta_{3}}{1+\beta_{3}}(4 \eta) i^{2} \operatorname{erf} c\left(\frac{\xi}{2 \sqrt{\eta}}\right)
\end{aligned}
$$

We have used the following Laplace inversion formulae [1]:

$$
\begin{gathered}
L^{-1}\left[\frac{e^{-a \sqrt{s}}}{s^{n / 2+1}}\right]=(4 \eta)^{n / 2} i^{n} \operatorname{erf} c\left(\frac{a}{2 \sqrt{\eta}}\right), \quad n=0,1,2, \ldots, \\
L^{-1}\left[\frac{e^{-a s}}{s^{n+1}}\right]=\frac{(\eta-a)^{n}}{\Gamma n+1} H(\eta-a), \quad n>-1,
\end{gathered}
$$

where the functions $\operatorname{erf}(x)$ and the associated complementary error functions of $n$th degree are defined by

$$
i^{n} \operatorname{erf} c(x)=\int_{x}^{\infty} i^{n-1} \operatorname{erf} c(\xi) d \xi, \quad n=1,2, \ldots
$$

with

$$
i^{0} \operatorname{erf} c(x)=\operatorname{erf} c(x)=\frac{2}{\sqrt{\pi}} \int_{x}^{\infty} e^{-u^{2}} d u, \quad \operatorname{erf} c(x)=1-\operatorname{erf}(x)
$$

\section{Numerical results and discussion}

The small-time solutions for displacement, temperature, stress, and the perturbed magnetic field in the solid reveal that each is composed of an elastic dilatational wave front traveling with unit dilatational speed and a diffusive part (thermal part of the solutions). The diffusive part arises due to the thermal damping term present in the heat transport equation. The diffusive part which is predominantly thermal propagates with infinite speed as expected. The terms associated with $H(\eta=\xi)$ represent the contribution of the wave traveling with unit speed at the wave front $\xi=\eta$ at time $t=\eta$. From solutions (2.49)-(2.51) and (2.53), we thus observed that the first part of the solutions represents a wave with exponential attenuation depending on the magneto-thermoelastic coupling $\varepsilon_{T}$. 

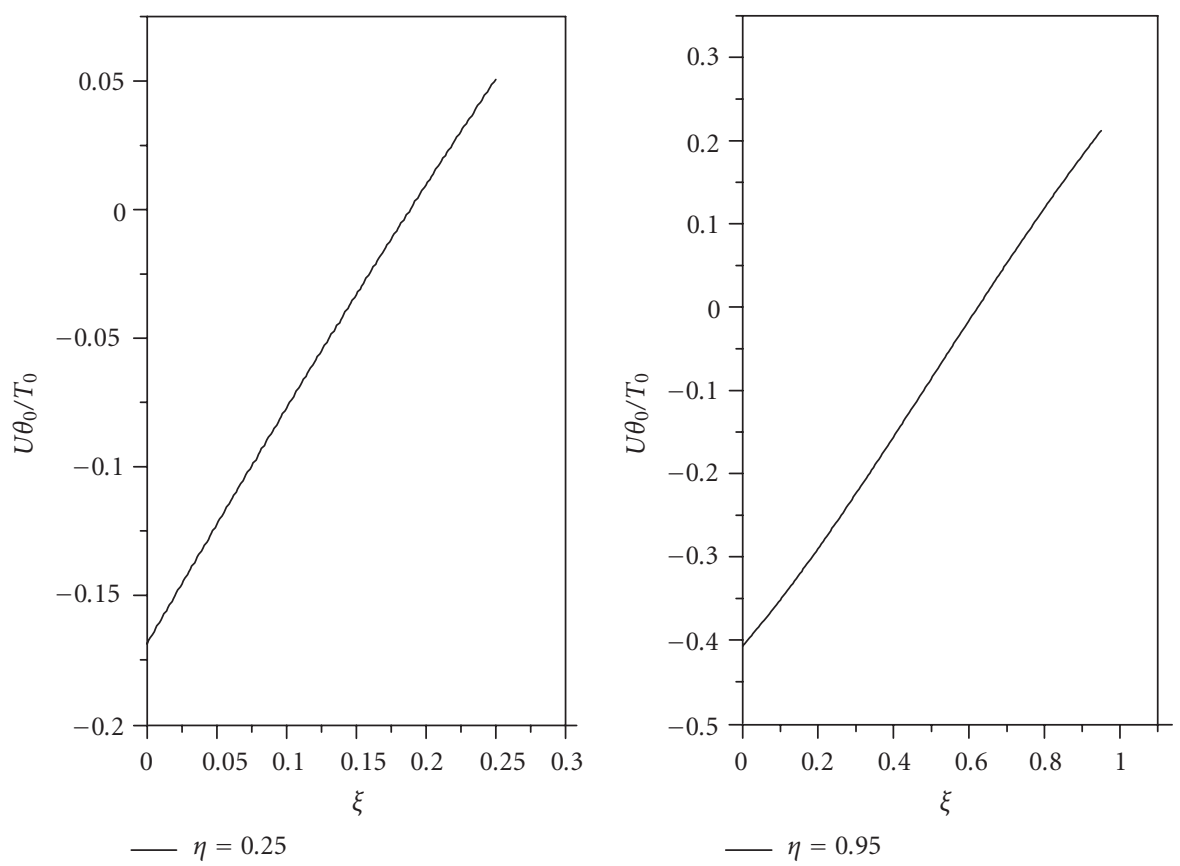

Figure 3.1. Displacement versus distance.

The displacement and temperature in the solid are both continuous at the elastic wave front while the stress and the perturbed magnetic field suffer finite discontinuities at this location. The discontinuities decay exponentially with distance from the boundary. The solution (2.52) for perturbed field in vacuum represents a wave propagating with Alfv'en acoustic wave $1 / \beta$ without any attenuation. Further, the perturbed field in vacuum is continuous at Alfv'en acoustic wave front. The finite discontinuities of the stress field and the perturbed magnetic field at the elastic wave front in the solid are not constants and are given by

$$
\begin{gathered}
{\left[\boldsymbol{\sigma}_{11}\right]_{\xi=\eta}=-\frac{T_{0}}{\theta_{0}} \frac{1}{1+\beta_{3}} \exp \left(-\frac{\varepsilon_{T}}{2} \xi\right), \quad \xi>0,} \\
{\left[h_{z}^{\prime}\right]_{\xi=\eta}=\frac{T_{0}}{\theta_{0}} \frac{1}{1+\beta_{3}} \exp \left(-\frac{\varepsilon_{T}}{2} \xi\right), \quad \xi>0 .}
\end{gathered}
$$

With an aim to illustrate the problem, we will present some numerical results. We have chosen a copper-like material for which $\varepsilon_{T}=0.0168, \beta_{3}=0.05$. We take $C_{T}=2$.

Using this data, the values of the physical quantities are evaluated as plotted in Figures $3.1,3.2,3.3$, and 3.4 .

Figure 3.1 represents variations of displacement against distance for two different times $\eta=0.25$ and $\eta=0.95$. It is observed that the displacement curve is continuous and it gradually increases with distance. The graph shows negative value in the range $0<\xi<0.188$ 
3316 Magneto-thermoelastic waves in thermoelasticity III

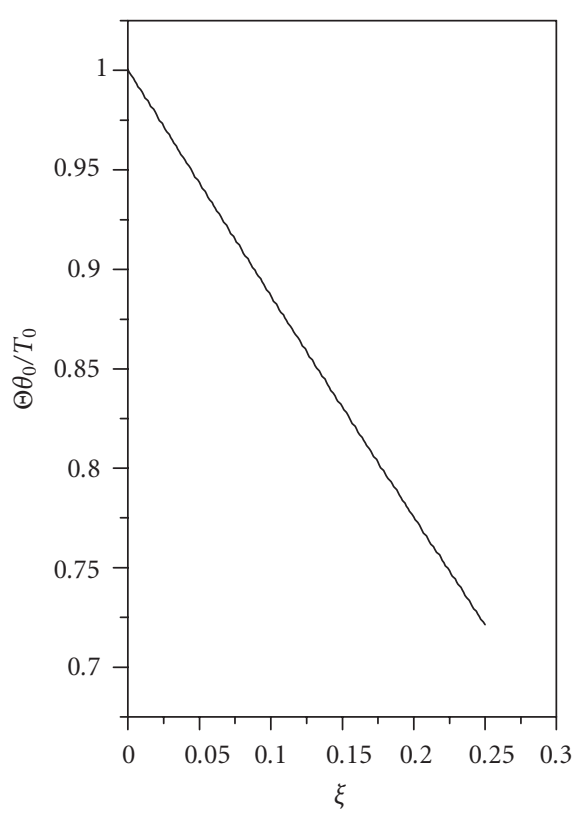

$\eta=0.25$

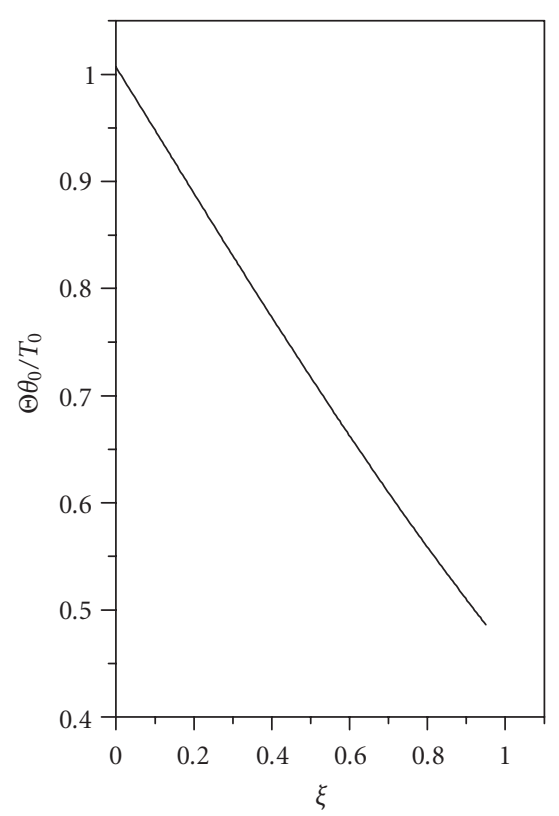

$\eta=0.95$

Figure 3.2. Temperature versus distance.
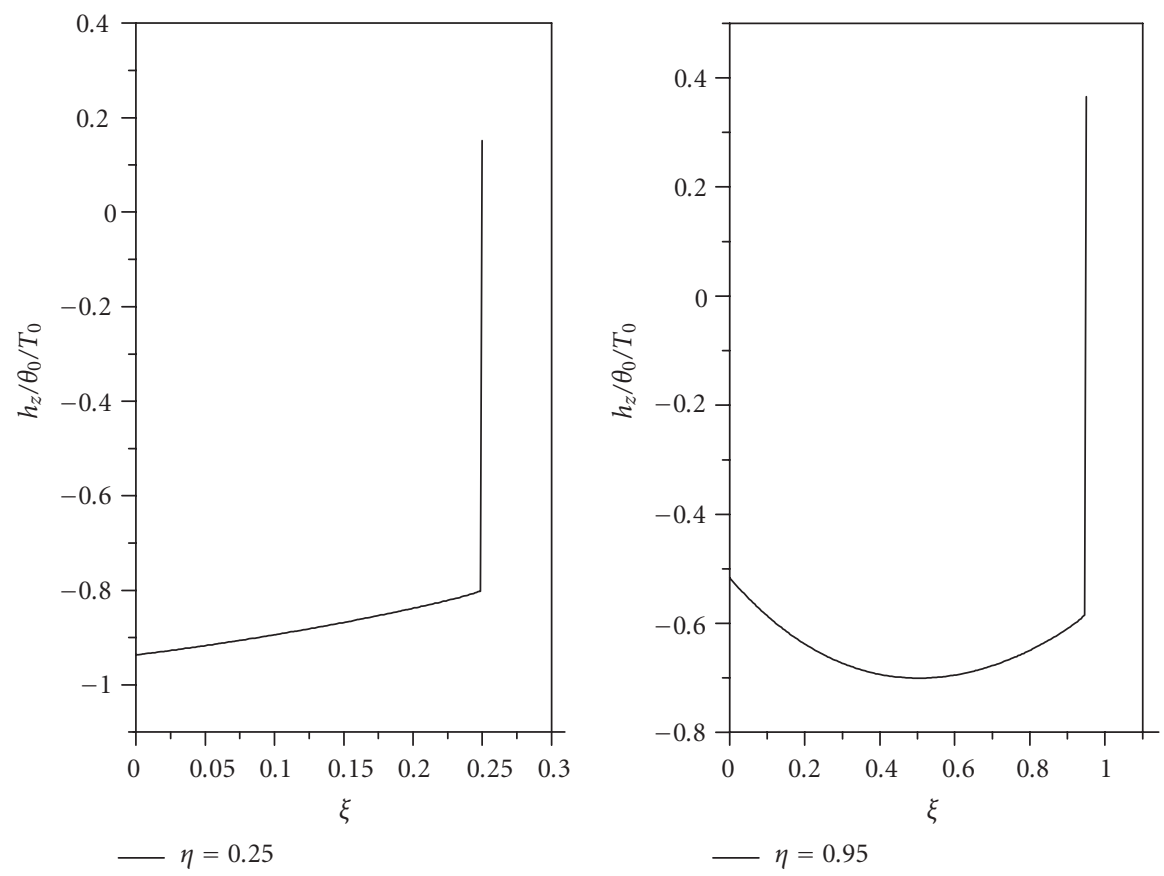

Figure 3.3. Perturbed field versus distance. 

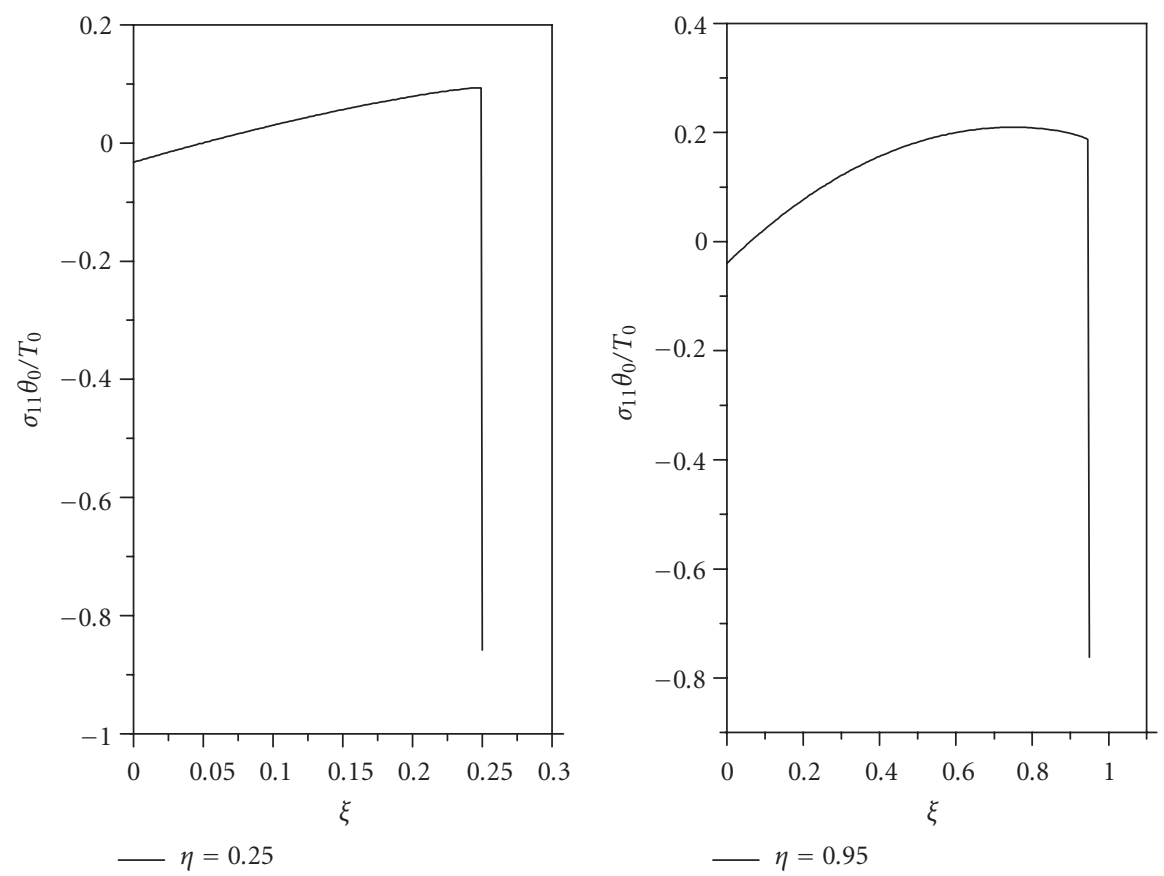

Figure 3.4. Stress versus distance.

Table 3.1

\begin{tabular}{ccc}
\hline Jumps & $\eta=0.25$ & $\eta=0.95$ \\
\hline$\left[\boldsymbol{\sigma}_{11} \theta_{0} / T_{0}\right]_{\xi=\eta}$ & -0.95170 & -0.94889 \\
{$\left[h_{z}^{\prime} \theta_{0} / T_{0}\right]_{\xi=\eta}$} & 0.95298 & 0.95032 \\
\hline
\end{tabular}

for $\eta=0.25$ and in the range $0<\xi<0.622$ for $\eta=0.95$, which means that it is in opposite direction.

Figure 3.2 indicates variation of temperature versus distance. The values of temperature gradually decrease with distance $\xi$, the curve is continuous in agreement with the theoretical results.

Figure 3.3 shows that the perturbed field gradually increases with distance for small time $\eta=0.25$ and suffers a finite jump at the elastic wave front $\xi=\eta=0.25$. Further for time $\eta=0.95$, the value of perturbed field first gradually increases with distance and then again it gradually decreases and suffers a finite jump at the elastic wave front $\xi=\eta=0.95$.

Figure 3.4 gives the stress distribution. Stress curve suffers a finite jump at two instants $\eta=0.25$ and $\eta=0.95$, where the wave front is positioned at the two instants $\eta=0.25$ and $\eta=0.95$ in agreement with theoretical results.

Finite jumps in stress and the perturbed magnetic fields at two different instants $\eta=$ 0.25 and $\eta=0.95$ are exhibited in Table 3.1.

The results are in complete agreement with the theoretical expression for jumps. 


\section{Magneto-thermoelastic waves in thermoelasticity III}

\section{Acknowledgment}

The authors thank the respected reviewers for their valuable suggestions.

\section{References}

[1] H. S. Carslaw and J. C. Jaeger, Conduction of Heat in Solids, 2nd ed., Clarendon Press, Oxford, 1959.

[2] D. Chand, J. N. Sharma, and S. P. Sud, Transient generalised magneto-thermo-elastic waves in a rotating half-space, Internat. J. Engrg. Sci. 28 (1990), no. 6, 547-556.

[3] G. Chatterjee (Roy) and S. K. Roychoudhuri, The coupled magneto-thermo-elastic problem in elastic half-space with two relaxation times, Internat. J. Engrg. Sci. 23 (1985), no. 9, 975986.

[4] A. E. Green and K. A. Lindsay, Thermo-elasticity, J. Elasticity 2 (1972), 1-7.

[5] A. E. Green and P. M. Naghdi, On undamped heat sources in an elastic solid, J. Thermal Stresses 15 (1992), 253-264.

[6] Thermoelasticity without energy dissipation, J. Elasticity 31 (1993), no. 3, 189-208.

[7] S. Kaliski and W. Nowacki, Combined elastic and electromagnetic waves produced by thermal shock in the case of a medium of finite electric conductivity, Bull. Acad. Polon. Sci. Sér. Sci. Tech. , 1, 10 (1962), 213-223.

[8] H. W. Lord and Y. Shulman, A generalized dynamical theory of thermoelasticity, J. Mech. Phys. Solids 15 (1967), 299-309.

[9] C. Massalas and A. Dalamangas, Coupled magneto-thermo-elastic problem in elastic half-space, Internat. J. Engrg. Sci. 21 (1983), no. 2, 171-178.

[10] Coupled magnetothermoelastic problem in elastic half-space having finite conductivity, Internat. J. Engrg. Sci. 21 (1983), no. 8, 991-999.

[11] W. Nowacki, Dynamical Problems of thermo-elasticity, Nordhoff, Layden, 1975.

[12] S. K. Roychoudhuri, Electro-magneto-thermo-elastic plane waves in rotating media with thermal relaxation, Internat. J. Engrg. Sci. 22 (1984), no. 5, 519-530.

[13] _ On magneto-thermo-elastic plane waves in infinite rotating media with thermal relaxation, Proc. IUTAM Symposium on "Electro-Magneto-Mechanical Interactions in Deformable Solids and Structures" (Y. Yamamoto and K. Miya, eds.), Elsevier, North Holland, 1986, pp. 361-366.

[14] S. K. Roychoudhuri and S. Banerjee, Magneto-thermo-elastic waves induced by a thermal shock in a finitely conducting elastic half space, Int. J. Math. Math. Sci. 19 (1996), no. 1, 131-143.

[15] S. K. Roychoudhuri and G. Chatterjee (Roy), A coupled magneto-thermoelastic problem in a perfectly conducting elastic half-space with thermal relaxation, Int. J. Math. Math. Sci. 13 (1990), no. 3, 567-578.

[16] - Temperature-rate dependent magneto- thermo-elastic waves in a finitely conducting elastic half-space, Comput. Math. Appl. 19 (1990), no. 5, 85-93.

[17] S. K. Roychoudhuri and L. Debnath, Magneto-thermo-elastic plane waves in rotating media, Internat. J. Engrg. Sci. 21 (1983), no. 2, 155-163.

S. K. Roychoudhuri: Department of Mathematics, University of Burdwan, Bardhaman 713104, West Bengal, India

E-mail address: skrc_bu_math@yahoo.com

Nupur Bandyopadhyay: Department of Mathematics, University of Burdwan, Bardhaman 713104, West Bengal, India

E-mail address: nupurbandyopadhyay@yahoo.co.in 


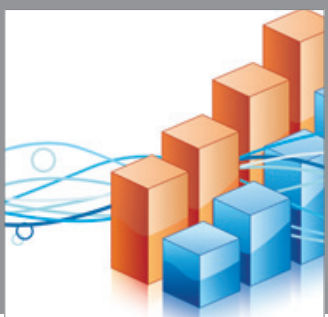

Advances in

Operations Research

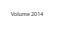

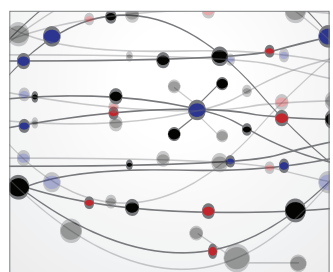

\section{The Scientific} World Journal
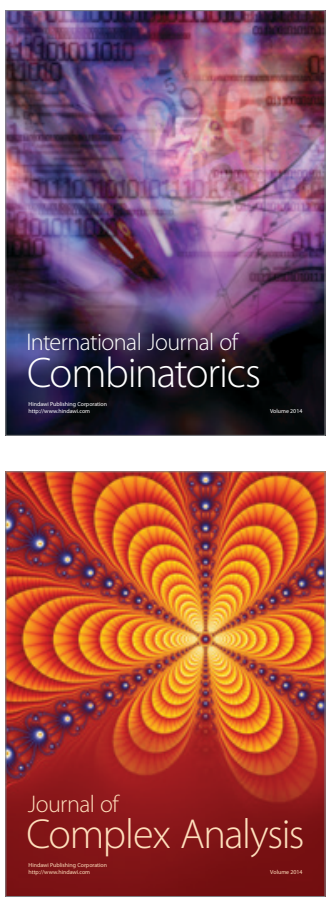

International Journal of

Mathematics and

Mathematical

Sciences
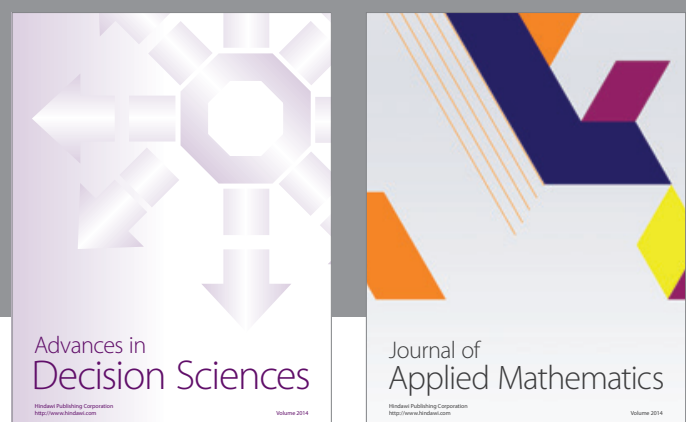

Journal of

Applied Mathematics
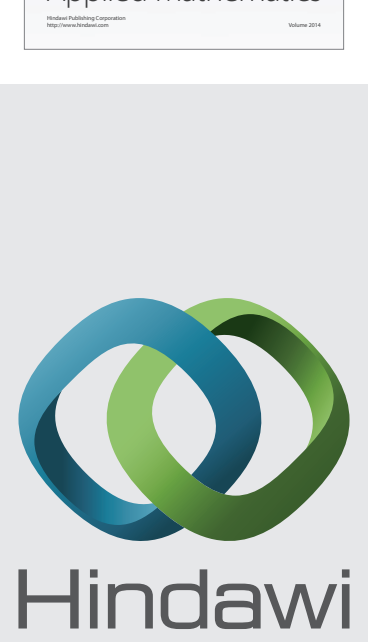

Submit your manuscripts at http://www.hindawi.com
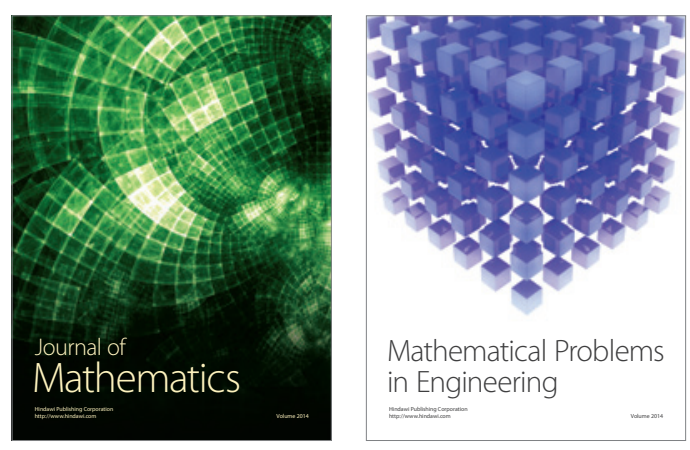

Mathematical Problems in Engineering
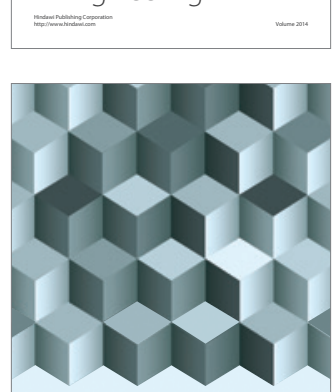

Journal of

Function Spaces
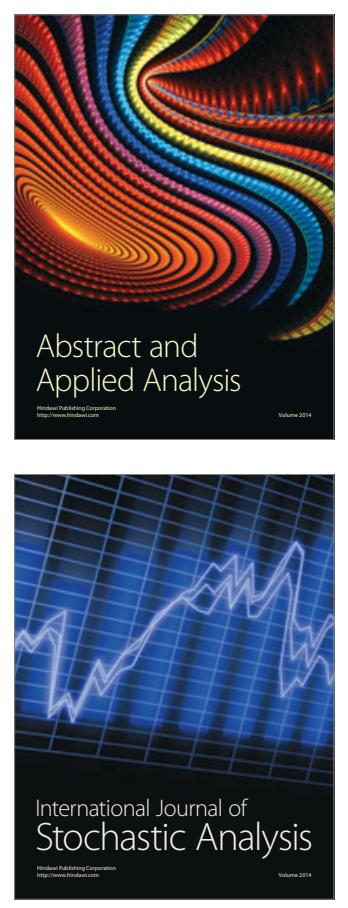

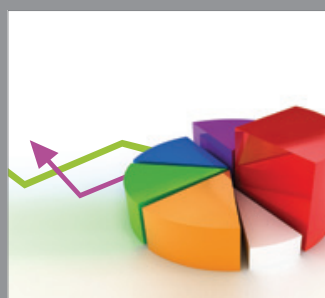

ournal of

Probability and Statistics

Promensencen
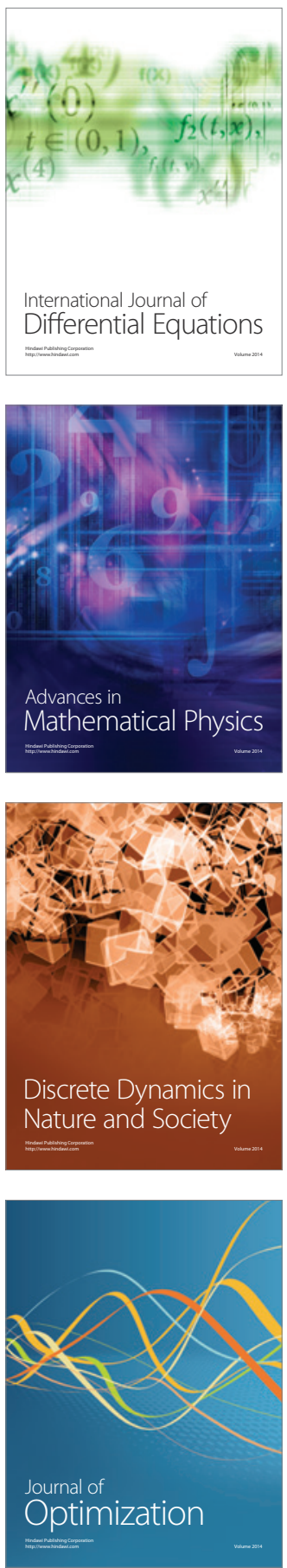